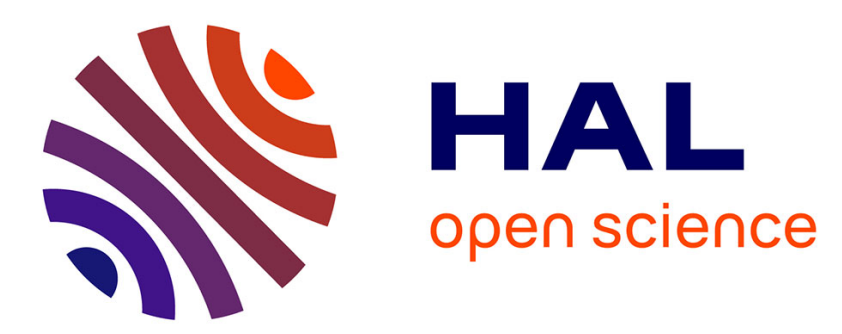

\title{
The eXtended Finite Element Method for cracked hyperelastic materials: a convergence study
}

\author{
A. Karoui, K. Mansouri, Y. Renard, M. Arfaoui
}

\section{To cite this version:}

A. Karoui, K. Mansouri, Y. Renard, M. Arfaoui. The eXtended Finite Element Method for cracked hyperelastic materials: a convergence study. International Journal for Numerical Methods in Engineering, 2014, 100 (3), pp.222-242. 10.1002/nme.4736 . hal-02072859

\section{HAL Id: hal-02072859 \\ https://hal.science/hal-02072859}

Submitted on 19 Mar 2019

HAL is a multi-disciplinary open access archive for the deposit and dissemination of scientific research documents, whether they are published or not. The documents may come from teaching and research institutions in France or abroad, or from public or private research centers.
L'archive ouverte pluridisciplinaire HAL, est destinée au dépôt et à la diffusion de documents scientifiques de niveau recherche, publiés ou non, émanant des établissements d'enseignement et de recherche français ou étrangers, des laboratoires publics ou privés. 


\title{
The eXtended Finite Element Method for cracked hyperelastic materials: a convergence study
}

\author{
A. Karoui ${ }^{1}$, K. Mansouri ${ }^{1,2}$, Y. Renard ${ }^{2 *}$ M. Arfaoui ${ }^{3}$
}

May 2, 2014

${ }^{1}$ Université de Tunis El Manar, École Nationale d'Ingénieurs de Tunis, LR-03-ES05

Laboratoire de Génie Civil, 1002, Tunis, Tunisie.

${ }^{2}$ Université de Lyon, CNRS, INSA-Lyon, ICJ UMR5208, LaMCoS UMR5259, F-69621,

Villeurbanne, France.

${ }^{3}$ Université de Tunis El Manar, École Nationale d'Ingénieurs de Tunis, LR-11-ES19

Laboratoire de Mécanique Appliquée et Ingénierie, 1002, Tunis, Tunisie.

\begin{abstract}
The present work aims to look into the contribution of the extended finite element method for large deformation of cracked bodies in plane strain approximation. The unavailability of sufficient mathematical tools and proofs for such problem makes the study exploratory. First, the asymptotic solution is presented. Then, a numerical analysis is realized to verify the pertinence of solution given by the asymptotic procedure, since it serves as an Xfem enrichment basis. finally, a convergence study is carried out to show the contribution of the exploitation of such method.
\end{abstract}

Key Words: Extended finite element method, hyperelastic material, cracktip, asymptotic displacement, large strain.

\section{Introduction}

The analysis of crack problems have long be based on the theory of linear elastic fracture mechanics (LEFM). Nevertheless, the contradiction between

\footnotetext{
${ }^{*}$ Correspondence to: Université de Lyon, CNRS, INSA-Lyon, ICJ UMR5208, LaMCoS UMR5259, F-69621, Villeurbanne, France, Yves.Renard@insa-lyon.fr
} 
the singular character of the displacement gradient near the crack tip and the assumptions of the linear theory, makes this last open to doubt. Other theories like nonlinear fracture mechanics are then developed to overcome this limitation, and more interesting results are found due to the emergence of numerical tools such as the finite element method, which enables the study of more complicated cases, analytically unresolved. In spite of its advantages, this method presents many drawbacks since its ability to detect singularities around the crack tip and geometrical discontinuities is very limited. One of the most classical strategies to bypass this constraint, is to refine the mesh, at least locally, and update it for time dependent problems, which makes computations long and expansive.

The eXtended Finite Element Method (Xfem), was introduced by Moës, Dolbow and Belytschko in $[23,24]$ to remove the need of minimal refinement, and improved later in [30], by the introduction of a technique to represent the geometry of the crack through some level set functions. Thanks to the capability of this method to incorporate analytical or sufficiently accurate numerical solutions as enrichment functions, it was widely employed to study singular phenomena, especially for nonlinear behavior. In [21], Legrain et al, used the Xfem to study a crack problem in an incompressible rubber-like material at large strain. Khoei et al, proposed in [16, 17] its application to treat contact and interfaces problems for two and three-dimensional large plasticity deformations, while Elguedj et al, in [12], employed it in order to study plastic fracture problems based on the Hutchinson-Rice-Rosengren (HRR) fields in the context of confined plasticity. Other applications of the method are mentioned in [13].

In the same way, the present study is based on the Xfem method and aims to analyze a crack problem for nonlinear (hyperelastic) material under large (plane) strain conditions. Two classical constitutive laws (Blatz-Ko and Ciarlet-Geymonat) will be used to test the convergence and the accuracy of the method. The enrichment to be considered is obtained from analytical analysis consisting to determine expressions of displacement and stress fields by means of an asymptotic procedure. In many bibliographic references (for instance $[19,20,29,31])$, it was shown that the local solution is independent of the domain geometry, which makes it valid for more general cases.

The first part of the present work, is devoted to present the asymptotic plane strain analysis of cracked hyperelastic compressible materials. The procedure is detailed for the Ciarlet-Geymonat material, whereas for the Blatz Ko material, only necessary results will be recalled from [19]. The second part is consecrated to present results obtained through the numerical implementation of the problem with Xfem, and a convergence study is then carried out. In particular, The sensitivity of the quality of the approximated 
solution with respect to the exponent of enrichment function is investigated.

\section{Asymptotic analysis of a crack tip problem in compressible hyperelastic materials}

\subsection{Formulation of a crack boundary value problem}

Consider an isotropic homogeneous compressible hyperelastic cracked body $\mathcal{B}$ which, in undeformed configuration, occupies an infinite cylindrical region $\mathcal{R}$ of the three-dimensional space $\mathbb{R}^{3}$ with

$$
\mathcal{R}=\left\{x \mid\left(x_{1}, x_{2}\right) \in \Omega, \quad-\infty<x_{3}<+\infty\right\},
$$

where $x$ is the position of a particle in the undeformed configuration and $\Omega$ denotes a cross section of $\mathcal{R}$ (Figure 1). The plane domain $\Omega$ of the twodimensional space $\mathbb{R}^{2}$, is described both in Euclidean coordinates and polar coordinates $r>0, \quad \theta \in[-\pi, \pi]$ relatively to the crack tip. Let us consider

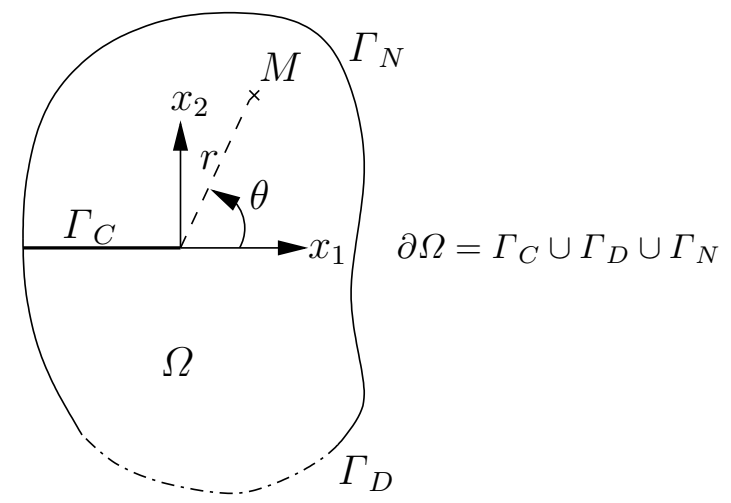

Figure 1: cross section $\Omega$ of the cracked domain in undeformed configuration

that the cylindrical body $\mathcal{B}$ is subjected to an invertible plane deformation, the position of a material point $x\left(x_{1}, x_{2}, x_{3}\right) \in \Omega$ is mapped to $y\left(y_{1}, y_{2}, y_{3}\right)$ on $\Omega^{*}$, with $\Omega^{*}$ the deformed representation of $\Omega$,

$$
y_{\alpha}(x)=x_{\alpha}+u_{\alpha}(x)(\alpha=1,2) \quad \forall x \in \Omega \quad \text { and } \quad y_{3}=x_{3},
$$

where $u(x)$ is the displacement vector. Assume that the mapping function $y \in \Omega$ is, at least, twice continuously differentiable on $\Omega$, i.e. $y \in C^{2}(\Omega)$, and then $u \in C^{2}(\Omega)$. To describe the geometry of deformation, the twodimensional deformation gradient $\boldsymbol{F}$ is introduced,

$$
\boldsymbol{F}(x)=\nabla y(x) \quad \Leftrightarrow \quad F_{\alpha \beta}=\frac{\partial y_{\alpha}}{\partial x_{\beta}}(\alpha, \beta=1,2) \quad \text { on } \Omega .
$$


$\boldsymbol{\nabla}($.$) is the gradient operator with respect to material coordinates.$

In order to guarantee that mapping $y$ performs a one-to-one continuously differentiable deformation, the associated deformation Jacobian $J$ (presenting the volume change) must be strictly positive

$$
0<J=\operatorname{det} \boldsymbol{F}=\lambda_{1} \lambda_{2}<+\infty \quad \text { on } \Omega .
$$

Here, $\lambda_{1}, \lambda_{2}, \lambda_{3}$ denote the principal stretches and $\lambda_{3}=1$ for plane deformation.

For hyperelastic isotropic compressible material, the existence of an elastic potential function $W$ per unit undeformed area is assumed,

$$
W(y(x))=W(\boldsymbol{F})=W(I, J),
$$

where the invariant $I$ is defined by:

$$
I=\operatorname{tr}\left(\boldsymbol{F}^{T} \boldsymbol{F}\right)=\lambda_{1}^{2}+\lambda_{2}^{2}>0 .
$$

The two-dimensional first Piola-Kirchhoff stress tensor $\boldsymbol{\tau}$ is written:

$$
\boldsymbol{\tau}=\frac{\partial W}{\partial \boldsymbol{F}}=J \frac{\partial W}{\partial J} \boldsymbol{F}^{-T}+2 \frac{\partial W}{\partial I} \boldsymbol{F} \quad \text { on } \Omega .
$$

The two-dimensional Cauchy stress tensor is then deduced,

$$
\boldsymbol{\sigma}=J^{-1} \boldsymbol{\tau} \boldsymbol{F}^{T}=J^{-1} \frac{\partial W}{\partial \boldsymbol{F}} \boldsymbol{F}^{T}=\frac{\partial W}{\partial J} \boldsymbol{I}+2 J^{-1} \frac{\partial W}{\partial I} \boldsymbol{F} \boldsymbol{F}^{T} \quad \text { on } \Omega^{*} .
$$

In absence of body forces, the strong form of the boundary value problem in undeformed configuration is expressed as follows:

$$
\left\{\begin{array}{l}
\operatorname{Div}(\boldsymbol{\tau})=0 \quad \text { on } \Omega \\
y(x)=y^{d} \quad \forall x \in \Gamma_{D} \\
t(x)=\boldsymbol{\tau} n=t^{n} \quad \forall x \in \Gamma_{N} \\
\boldsymbol{\tau} n=0 \quad \forall x \in \Gamma_{C} .
\end{array}\right.
$$

Where $\operatorname{Div}($.$) is the divergence operator with respect to material coordinates.$ The boundary $\partial \Omega$ of the cracked body $\mathcal{B}$ is partitioned into Dirichlet boundary $\Gamma_{D}$, Neumann boundary $\Gamma_{N}$ and crack face boundary $\Gamma_{C}$. The vector $n$ denotes the unit normal vector to the boundary in the undeformed configuration, while $y^{d}$ and $t^{n}$ denote the prescribed deformation and traction vectors in the undeformed configuration, respectively. This last one be characterized by a combination of modes I and II loadings conditions [29]). 
Solving the local crack problem is a quite complicated problem (see [27]). In this case, the deformation $y$ is supposed to belong to the set of admissible deformations with finite potential energy,

$$
\mathcal{C}=\left\{y(x) \mid x \in \Omega, J=\operatorname{det}(\boldsymbol{F})>0, y(x)=y^{d} \text { on } \Gamma_{D}, E_{\text {pot }}<+\infty\right\},
$$

where the potential energy functional $E_{\text {pot }}(y(x))$ is defined by:

$$
E_{p o t}=\int_{\Omega_{0}} W(y(x)) d \Omega-\int_{\Gamma_{N}} t^{d} y d \Gamma<+\infty .
$$

Condition (1.11) restricts the nature of singularity of the deformation gradient $\boldsymbol{F}$ near the crack tip, which is due to the body geometrical configuration $\mathcal{B}$. Solving the above boundary value problem is equivalent to find the minimizer point of the potential energy functional (1.11), when deformation belongs to the set of admissible deformations $\mathcal{C}$.

Finally, let $\Im$ be the class of all $\{y, \boldsymbol{\sigma}, J\}$ satisfying the boundary value problem. Thus, it is easy to prove that

$$
\{y, \sigma, J\} \subset \Im \Leftrightarrow\left\{\boldsymbol{Q} y, \boldsymbol{Q} \boldsymbol{\sigma} \boldsymbol{Q}^{T}, J\right\} \subset \Im, \forall \boldsymbol{Q} \text { a proper order tensor }
$$

This is ensured by the objectivity of the constitutive equation and by the form of the boundary conditions. This property will be used later on to better understand the nature of the local transformation field [29].

\subsection{Singular elastostatic field near the crack tips for a Ciarlet-Geymonat hyperelastic material}

\subsubsection{Constitutive equations}

In this section, The analysis is devoted to the so called Ciarlet-Geymonat hyperelastic material $[8,14]$. Such hyperelastic potential is polyconvex, and satisfies coerciveness inequality, which is an essential tool in existence theorems $[2,8]$. For the plane deformation case, this potential takes the following form:

$$
W_{1}(I, J)=A_{1}(I-2)+B_{1}\left(I+J^{2}-3\right)+\Gamma(J) .
$$

The function $\Gamma$ is defined by

$$
\Gamma: \delta>0 \rightarrow \Gamma(\delta)=C_{1}\left(\delta^{2}-1\right)-D_{1} \log (\delta) .
$$

In order to ensure the convexity of this function, parameters $A_{1}, B_{1}, C_{1}$ and $D_{1}$ must verify the following conditions (see [8]):

$$
\operatorname{Max}\left(0, \frac{\mu}{2}-\frac{\lambda}{4}\right)<A_{1}<\frac{\mu}{2}, B_{1}=\frac{\mu}{2}-A_{1}, C_{1}=\frac{\lambda}{4}-\frac{\mu}{2}+A_{1}, \text { and } D_{1}=\mu+\frac{\lambda}{2},
$$


where $\lambda>0$ and $\mu>0$ are Lamé coefficients.

The comprehension of material behavior when subjected to a pure homogeneous plane deformation, is necessary for our purpose, before the asymptotic formulation of the problem (cf. Knowles and Sternberg [19] and Le and Stumpf [20]). Thus, consider a state of uni-axial tension parallel to the $x_{2}$-axis. The transverse stretch is then parallel to the $x_{1}$-axis,

$$
\begin{gathered}
y_{i}=\lambda_{i} x_{i},(i=1,2) \quad(\text { no sum }), \\
\lambda_{2}=\lambda>1 \text { and } \lambda_{1}=\bar{\lambda}(\lambda),
\end{gathered}
$$

The stress state corresponding to such deformation is,

$$
\sigma_{i j}=0(i \neq j), \sigma_{11}=0, \sigma_{22}=\sigma_{22}(\bar{\lambda}, \lambda) \text {, on } \Omega^{*} .
$$

From (1.8), (1.16) and (1.17), one can easily deduce:

$$
\sigma_{11}=\frac{1}{\lambda_{2}} \frac{\partial W_{1}}{\partial \lambda_{1}}=0, \text { on } \Omega^{*}
$$

To determine how $\bar{\lambda}(\lambda)$ behaves asymptotically as $\lambda \rightarrow \infty$, we take $\lambda_{2}=\lambda$ and $\lambda_{1}=\bar{\lambda}(\lambda)$ in (1.16). Then, by proceeding to the limit and after keeping only dominant terms, equation (1.18) gives

$$
\bar{\lambda}(\lambda)=\lambda^{-1}\left[\frac{D_{1}}{2\left(B_{1}+C_{1}\right)}\right]^{\frac{1}{2}}+o\left(\lambda^{-1}\right) \quad \text { as } \lambda \rightarrow \infty .
$$

Accordingly, the transformation Jacobian $J$ takes the form:

$$
J=\lambda_{1} \lambda_{2}=\lambda \bar{\lambda}=\left[\frac{D_{1}}{2\left(B_{1}+C_{1}\right)}\right]^{\frac{1}{2}}+o(1) .
$$

According to this result, we can conclude that $J$ remains constant as $\lambda \rightarrow \infty$. This property depends on material behavior through the elastic potential $W$ $[18,20]$.

\subsubsection{First order asymptotic analysis}

Our main objective is to resolve the plane strain problem stated through (1.9) for a class of hyperelastic materials whose strain energy is given by (1.13). Then, we assume that solution corresponding to such problem admits the asymptotic representation:

$$
\begin{gathered}
y_{i}(r, \theta)=r^{m_{1}} u_{i}(\theta)+o\left(r^{m_{1}}\right),(i=1,2), \quad-\pi<\theta<\pi, \\
0<m_{1}<1,
\end{gathered}
$$


where the condition on $m_{1}$ guarantees that transformation $y_{i}$ remains bounded while stresses become singular near the crack tip. Functions $u_{1}$ and $u_{2}$ must be, at least, twice continuously differentiable and fail to vanish identically on $[-\pi, \pi]$.

The asymptotic form of the two deformation invariants is established from the combination of (1.4), (1.6) and (1.21),

$$
\begin{gathered}
I=r^{2\left(m_{1}-1\right)} p(\theta)+o\left(r^{2\left(m_{1}-1\right)}\right), \quad p(\theta)=m_{1}^{2}\left(u_{1}^{2}+u_{2}^{2}\right)+\left(\dot{u}_{1}^{2}+\dot{u}_{2}^{2}\right), \\
J=r^{2\left(m_{1}-1\right)} q(\theta)+o\left(r^{2\left(m_{1}-1\right)}\right), \quad q(\theta)=m_{1}\left(u_{1} \dot{u}_{2}-u_{2} \dot{u}_{1}\right) .
\end{gathered}
$$

The coefficient $q(\theta)$ relative to the first order expression of $J$ can vanish identically on $[-\pi, \pi]$. It is then possible that the first non-zero coefficient may appear at a higher order of $r$. According to this remark, we will write

$$
J=r^{l_{1}} H_{1}(\theta)+o\left(r^{l_{1}}\right), \quad H_{1}(\theta)>0 .
$$

The comparison between the two expressions of $J$, i.e. (1.23) and the second of (1.22), leads to

$$
2\left(m_{1}-1\right) \leq l_{1}
$$

Indeed, if $l_{1}<2\left(m_{1}-1\right)$, then $H_{1}(\theta)=0$, which contradicts (1.23). In order to determine parameter $l_{1}$ and function $H_{1}$, we invoke results given in the case of pure homogeneous plane deformation, and we assume that if $\lambda \rightarrow \infty$, we have a local state of uni-axial traction. Consequently, the identification between (1.20) and (1.23) for $\theta= \pm \pi$ gives

$$
H_{1}=\left[\frac{D_{1}}{2\left(B_{1}+C_{1}\right)}\right]^{\frac{1}{2}}, \quad l_{1}=0 .
$$

Inequality (1.24) transforms through condition $m_{1}<1$ to $2\left(m_{1}-1\right)<l_{1}$. Hence, we have $q(\theta)=m_{1}\left(u_{1} \dot{u}_{2}-u_{2} \dot{u}_{1}\right)=0$. The solution of such equation is of the form:

$$
u_{i}=a_{i} U(\theta), U \neq 0, \quad(i=1,2) .
$$

In order to determine $U(\theta)$, we proceed to the resolution of equilibrium equations given in (1.9), which by means of (1.7), (1.3) and (1.13) leads to

$$
\frac{\partial W_{1}}{\partial I} \Delta y_{i}=0,(i=1,2) \Rightarrow m_{1}^{2} u+\ddot{u}=0 .
$$

The corresponding solution is given by

$$
U(\theta)=b_{1} \sin \left(m_{1} \theta\right)+b_{2} \cos \left(m_{1} \theta\right) ; \quad b_{1}, b_{2} \in \mathbb{R} .
$$


Boundary conditions given in (1.9), together with (1.7), (1.13) and (1.20) furnish:

$$
\left(a_{1}^{2}+a_{2}^{2}\right) U( \pm \pi) \dot{U}( \pm \pi)=0 .
$$

Therefore, three cases arise: $U( \pm \pi)=0$, both $U( \pm \pi)$ and $\dot{U}( \pm \pi)$ vanishes and $\dot{U}( \pm \pi)=0$. The first case means that all points on the crack edge map the crack tip, which is meaningless from a physical point of view. The second case implies, through (1.22) that $I=0$ for $\theta= \pm \pi$, which is impossible since $I=\lambda_{1}^{2}+\lambda_{2}^{2}>0\left(\lambda_{i}>0\right)$. As a result, only the case $\dot{U}( \pm \pi)=0$ holds. Thus, this result together with (1.28) provide the problem global solution

$$
\begin{gathered}
y_{i}(r, \theta)=a_{i} r^{m_{1}} U(\theta)+o\left(r^{m_{1}}\right),(i=1,2), \quad-\pi<\theta<\pi, \\
m_{1}=\frac{1}{2}, \text { and } U(\theta)=\sin \left(m_{1} \theta\right) .
\end{gathered}
$$

Now, we recall the objectivity principal, especially property given by (1.12), and with a special choice of the proper orthogonal tensor $\boldsymbol{Q}$ (corresponding to a rigid body motion), we obtain:

$$
\left[y_{i}^{*}\right]=\left[Q_{i j}\right]\left[y_{j}\right], \quad\left[Q_{i j}\right]=\left[\begin{array}{rr}
\frac{a_{2}}{a} & -\frac{a_{1}}{a} \\
\frac{a_{1}}{a} & \frac{a_{2}}{a}
\end{array}\right] \quad \text { and } a=a_{1}^{2}+a_{2}^{2},
$$

then, we deduce

$$
\left\{\begin{array}{l}
y_{1}^{*}=o\left(r^{m_{1}}\right) \\
y_{2}^{*}=a \sin \left(m_{1} \theta\right)+o\left(r^{m_{1}}\right)
\end{array}\right.
$$

Such solution provides the following weak estimate:

$$
J \sim o\left(r^{-1}\right)
$$

which presents a number of mathematical and physical inconsistencies and is therefore inadequate. In fact, the Jacobian $J$ has a degenerate form which reflects the degenerate character of the deformation asymptotic approximation (1.21) which is not locally one-to-one.

\subsubsection{Second order asymptotic analysis}

The first order approximation to the local deformation in the vicinity of the crack tips does not constitute an invertible mapping. Consequently, we must refine (1.21) and (1.22) by developing a two term approximation,

$$
\begin{gathered}
y_{\alpha}=a_{\alpha} r^{m_{1}} U(\theta)+r^{m_{2}} V_{\alpha}(\theta)+o\left(r^{m_{2}}\right), V_{\alpha}(\theta) \in C^{2}([-\pi, \pi]), V_{\alpha} \neq 0 \\
J(r, \theta)=H_{1}+r^{l_{2}}+o\left(r^{l_{2}}\right), H_{2}(\theta) \in C^{1}([-\pi, \pi]), H_{2} \neq 0
\end{gathered}
$$


with $m_{2}>m_{1}, l_{2}>0, V_{\alpha}(\theta)$ and $H_{2}(\theta)$ are still undetermined, whereas $m_{1}, U$ and $H_{1}$ are now given by (1.25) and (1.30). Using the asymptotic deformation form (1.34), the deformation invariants $J$ and $I$ become

$$
\begin{gathered}
J=r^{m_{1}+m_{2}-2}\left(m_{1} U \dot{\Psi}_{2}+m_{2} \dot{U} \Psi_{2}\right)+o\left(r^{m_{1}+m_{2}-2}\right) \quad \text { on }[-\pi, \pi], \\
I=a^{2} r^{2\left(m_{1}-1\right)} G(\theta)+r^{\left(m_{1}+m_{2}-2\right)} K(\theta)+o\left(r^{\left(m_{1}+m_{2}-2\right)}\right) \quad \text { on }[-\pi, \pi],
\end{gathered}
$$

where

$$
\left\{\begin{array}{l}
G(\theta)=\dot{U}^{2}(\theta)+m_{1} U^{2}(\theta) \\
K(\theta)=m_{1} m_{2} U(\theta) \chi_{2}(\theta)+\dot{U}(\theta) \dot{\chi}_{2}(\theta) \\
\chi_{2}(\theta)=a_{1} V_{1}(\theta)+a_{2} V_{2}(\theta) \\
\Psi_{2}(\theta)=a_{1} V_{2}(\theta)-a_{2} V_{1}(\theta)
\end{array}\right.
$$

Comparing the Jacobian expressions given by (1.35) and (1.36), one can deduce that $m_{1}+m_{2}-2 \leq 0$. Consequently

$$
\begin{array}{ll}
m_{1} U \dot{\Psi}_{2}-m_{2} \dot{U} \Psi_{2}=0 & \text { on }[-\pi, \pi] \quad \text { if } \quad m_{1}<m_{2}<2-m_{1}, \\
m_{1} U \dot{\Psi}_{2}-m_{2} \dot{U} \Psi_{2}=0 & \text { on }[-\pi, \pi] \text { if } \quad m_{1}<m_{2}=2-m_{1} .
\end{array}
$$

Boundary conditions can be obtained from (1.39) and (1.40),

$$
\begin{gathered}
\dot{\Psi}_{2}( \pm \omega)=0 \quad \text { if } \quad m_{1}<m_{2}<2-m_{1} \\
\dot{\Psi}_{2}( \pm \omega)=\frac{H_{1}}{m_{1} U( \pm \omega)} \quad \text { if } \quad m_{2}=2-m_{1} .
\end{gathered}
$$

These boundary conditions are not natural and do not have physical significance. They come from the first order differential equations (1.39) and (1.40). In order to obtain other conditions for the function $\Psi_{2}(\theta)$, we recall that equilibrium equation is strongly elliptic due to the polyconvexity of the hyperelastic potential $W_{1}$, then the associated boundary value problem solution has continuous partial derivatives for all orders. So, $\Psi_{2}(\theta)$ is $C^{\infty}([-\pi, \pi])$ (cf. $\left.[19,20,29]\right)$. After replacing (1.34) and (1.35) in the equilibrium field equations and the traction free boundary conditions (1.9), then recalling that $U$ satisfies relation (1.27), one obtains the eigenvalue problem

$$
\left\{\begin{array}{l}
\ddot{\chi}_{2}+m_{2} \chi_{2}=0 \\
\dot{\chi_{2}}( \pm \pi)=0
\end{array} \text { on }[-\pi, \pi], m_{1}<m_{2} \leq 2-m_{1} .\right.
$$

The two eigenvalue problems are now well defined for $\Psi$ and $\chi$, with $m_{2}$ an eigenvalue parameter whose minimal value will be considered. 
The solution of the eigenvalue problem on $\chi_{2}$, defined by (1.43) with $m_{2}$ as parameter is given by:

$$
\chi_{2}(\theta)=b_{1} \cos \left(m_{2} \theta\right) \text { on }[-\pi, \pi],
$$

while (1.39) with condition $\Psi_{2}$ in $C^{\infty}$ gives

$$
\Psi_{2}(\theta)=b_{2} U^{2}(\theta)=b_{2} \sin ^{2}\left(m_{1} \theta\right) \quad \text { on }[-\pi, \pi] \text { and } m_{2}=2 m_{1}=1,
$$

where $b_{2}$ is a real constant.

In the same way, equilibrium equations and boundary conditions furnish the eigenvalue problem on $J$ :

$$
\begin{gathered}
l_{2}=m_{1}=\frac{1}{2} \\
4\left(A_{1}+B_{1}\right)\left(\ddot{\Psi}_{2}+m_{2}^{2} \Psi_{2}\right)+a^{2} \Lambda_{2}\left(m_{1} \dot{H}_{2} U-l_{2} H_{2} \dot{U}\right)=0 \quad \text { on }[-\pi, \pi] \\
4\left(A_{1}+B_{1}\right) \dot{\Psi}_{2}+a^{2} \Lambda_{2} H_{2} U=0 \quad \text { at } \quad \theta= \pm \pi
\end{gathered}
$$

where, $\Lambda_{2}=2\left(B_{1}+C_{1}\right)-\frac{D_{1}}{H_{1}^{2}}$.

By combining (1.45), (1.47) and (1.48), one arrives to:

$$
H_{2}(\theta)=\frac{4\left(A_{1}+B_{1}\right) b_{2}}{\Lambda_{2} a^{2}} \cos \left(l_{2} \theta\right)
$$

With a similar analysis to the one developed for the first order asymptotic procedure, we find that, again, the deformation asymptotic development (1.34) provides a weak estimate of the deformation Jacobian $J$. However, we do not need to refine our approximation by a third order asymptotic approximation, since it will not be necessary for the Xfem enrichment.

\subsection{Singular elastostatic field near the crack tips for a Blatz-Ko material}

This strain energy function was introduced by Blatz and Ko in [4] to model a highly compressible rubber-like material behavior. Knowles and Sternberg proposed a corrected form [19]

$$
W_{2}(I, J)=\left(A_{2} I+B_{2} J+C_{2} \frac{I}{J^{2}}+D_{2}\right)^{n},
$$

where $A_{2}, B_{2}, C_{2}, D_{2}$ are constants depending on material and $n$ is a hardening parameter. 
In order to satisfy the Baker-Ericksen inequality and the Noll-Coleman requirement [1, 9, 10], Knowles and Sternberg showed that material parameters must verify the following inequalities:

$$
A_{2}>0, \quad 0<B_{2}<2 A_{2}, \quad C_{2}>0 \quad \text { and } \quad \frac{1}{2}<n<\infty .
$$

An asymptotic procedure is developed in [19] in order to resolve a problem similar to that enunciated in the previous section. Only singular part of the transformation is given here, where the entirely solution is detailed in [19]:

$$
y_{2}(r, \theta)=r^{1-\frac{1}{2 n}} f(\theta), \quad(-\pi \leq \theta \leq \pi),
$$

$f(\theta)$ being a function depending on $\theta$. It admits the following form:

$$
\begin{aligned}
f(\theta)= & d_{1} \sin \frac{\theta}{2}\left[1-\frac{2 k^{2} \cos ^{2}\left(\frac{\theta}{2}\right)}{1+\omega(\theta, n)}\right]^{1 / 2}[\omega(\theta, n)+k \cos \theta]^{k / 2}, \\
& \omega(\theta, n)=\left[1-k \sin ^{2} \theta\right]^{1 / 2} \text { and } k=\frac{n-1}{n},
\end{aligned}
$$

where, $d_{1}$ designates a constant depending on boundary conditions at infinity.

The singular part of the transformation for Ciarlet-Geymonat material, relatively to the first order asymptotic development, is of the form $y_{2}(r, \theta)=$ $r^{\frac{1}{2}} a_{2} \sin \left(\frac{\theta}{2}\right)$. Now, let's remark that, when the Blatz-Ko material parameter $n$ is equal to 1 , the singular transformation for both two materials have the same asymptotic form, which is given by

$$
y_{2}(r, \theta)=r^{\frac{1}{2}} \sin \left(\frac{\theta}{2}\right), \quad(\alpha=1,2),
$$

which constitutes a unique Xfem enrichment basis.

\section{The Xfem cut-off Method}

Many mechanical problems are related to discontinuous geometries (crack, vertex, hole etc.), which leads in most cases to the presence of singularities (when stress and strain become unbounded). Therefore, the analysis of such problems by means of the classical finite element method requires some specific precautions, like mesh refinement and mesh update (for time dependent problems), which increases computation time and cost.

The Xfem method (eXtended Finite Element Method) was introduced by Moës et al in [23], and became rapidly an important element of modeling in a wide domain of applications due to its interesting advantages. Indeed, it makes possible the decoupling of mesh and geometrical discontinuities, 


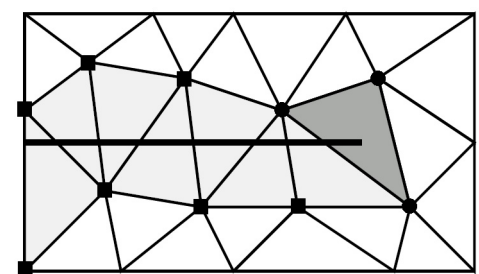

- Singular enrichment

- Heaviside enrichment

Figure 2: Xfem enrichment

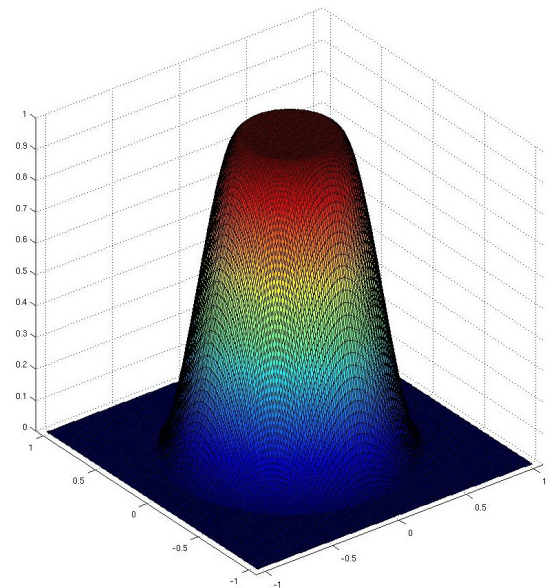

Figure 3: Cut-off function

and in contrast to other methods like Generalized Finite Element Method or Partition of Unity Finite Element Method, which adopt a global enrichment (see [25]), the Xfem enrichment is realized at a local level.

The crack (or geometrical discontinuity in general) is taken into account, within Xfem framework, by recourse to the following step (or Heaviside like) function, taking into account the displacement jump between the two sides of the crack:

$$
H(x)= \begin{cases}1 & \text { for }\left(x-x^{c}\right) \cdot n>0 \\ -1 & \text { elsewhere }\end{cases}
$$

where, $x^{c}$ denotes the crack position and $n$ the unit outward normal vector to the crack face.

This enrichment concerns nodes whose corresponding shape functions supports are entirely cut by the crack (see Figure 2), while nodes of convex containing the crack tip are enriched by the singular functions basis obtained from asymptotic analysis. For both Ciarlet-Geymonat and Blatz-Ko cases ( $n$ taken equal to 1 for the second case), this basis takes the form

$$
\{F(x)\}=\left\{r^{1 / 2} \sin \left(\frac{\theta}{2}\right)\right\},
$$

The Xfem cut-off variant, enables finding satisfactory results, without increasing outstandingly the number of degrees of freedom or deteriorating the associated linear system condition number. Besides, it consists to make a regular transition between enriched and non-enriched regions. Then, this variant avoids limitations met in the case of others variants like Xfem with fixed enrichment area (see [6]). Note that there exists some other methods proposed in literature to resolve the conditioning problem inherent to the asymptotic 
enrichment. In [3], the Xfem implementation was improved by mean of an additional preconditioning based on a local Cholesky decomposition. In [7], authors showed the significant contribution of a new strategy of vectorial enrichment to the improvement of convergence rates and condition number, in the context of linear fracture mechanics. In [22], a corrected (or a modified) extended finite element method was proposed for three-dimensional problems with some remedies for limitations caused by the linearly dependence of the enrichment functions to the blending elements.

The singular enrichment is realized in a region around the crack tip, according to a cut-off function $\chi$ (Figure 3 ), defined by two parameters $r_{0}$ and $r_{1}\left(r_{0}<r_{1}\right)$, such that

$$
\begin{cases}\chi(r)=1 & \text { if } r<r_{0}, \\ 0<\chi(r)<1 & \text { if } r_{0}<r<r_{1}, \\ \chi(r)=0 & \text { if } r>r_{1}\end{cases}
$$

Consequently, the Xfem cut-off enriched space has the following form:

$$
V^{h}=\left\{v^{h} ; v^{h}=\sum_{i \in I} a_{i} \varphi_{i}+\sum_{i \in I_{H}} b_{i} H \varphi_{i}+c F \chi ; a_{i}, b_{i}, c \in \mathbb{R}^{2}\right\},
$$

where, the three terms designate, successively, the classical finite element method term, the Heaviside enrichment term and finally the singular enrichment term ( $I$ being the set of all finite element node indices and $I_{H}$ the set of node indices corresponding to the finite element shape functions $\varphi_{i}$ having their support entirely cut by the crack).

\section{$3 \quad$ Numerical tests}

For numerical tests, we consider a non-cracked domain $\bar{\Omega}$ being a square defined by:

$$
\bar{\Omega}=[-0.5,0.5] \times[-0.5,0.5] .
$$

The crack curve is designated by $\Gamma_{C}=[-0.5,0] \times\{0\}$ (see Figure 4 ). The cut-off function is chosen independent of $\theta$ and being the unique $C^{2}(0,+\infty)$ piecewise fifth-order polynomial in $r$ verifying

$$
\begin{cases}\chi(r)=1 & \text { if } r<0.06 \\ 0<\chi(r)<1 & \text { if } 0.06<r<0.35 \\ \chi(r)=0 & \text { if } r>0.35\end{cases}
$$

The stress state to be considered is an opening mode of the crack. Neumann condition are introduced by a symmetric linear traction forces applied 


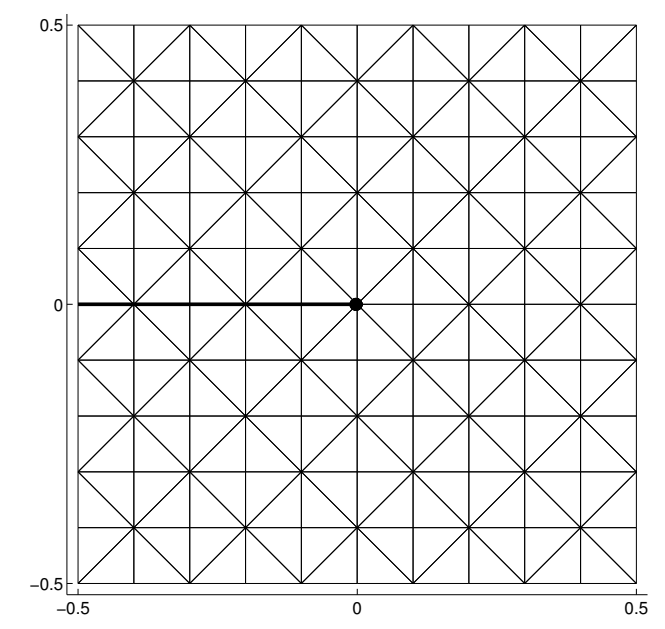

Figure 4: The triangulation of the non-cracked domain

on the upper and lower boundaries. In order to avoid singular phenomena which can arise on the Dirichlet-Neumann transition and may perturb the analysis, only a Neumann boundary condition is considered. The lateral boundaries are traction free and to prevent rigid body motions the displacement is prescribed on two points in the horizontal axis of symmetry. At the first point $(0.5,0)$, vertical and horizontal translations are eliminated, and to prevent rotations, vertical translation are eliminated in the second point $(0.2,0)$.

The choice of parameters defining the two materials must satisfy the conditions (1.15) and (1.51). Accordingly, we take for all tests, the above values:

$$
A_{1}=B_{1}=1, C_{1}=3 / 2, D_{1}=2, n=1, \lambda=\mu=1 \text { and } \gamma=0.3,
$$

while applied forces $F$ measure $\left\|F_{B K}\right\|=2$ for the Blatz-Ko material and $\left\|F_{C G}\right\|=0.5$ for the Ciarlet-Geymonat material (all physical quantities are expressed in the international system of units). Then, they will be reduced to one per cent for some tests at small deformations. These tests are considered in order to make a comparison with the linear theory and do not mean that singularity disappears (it is still present at the crack-tip even in the case of linear theory).

Now, since exact solution for such problem is not analytically known, the considered reference solution was obtained by Xfem cut-off method and by mean of Lagrange elements $P_{K+1}$ with a very fine mesh (while the approximated solution is then obtained by means of $P_{K}$ elements). 
Figures 5 and 6 show the numerical solution obtained by Xfem method. The displacement and Von-Mises stress fields distribution are presented for Blatz-Ko material (a similar form is obtained for Ciarlet-Geymonat material).

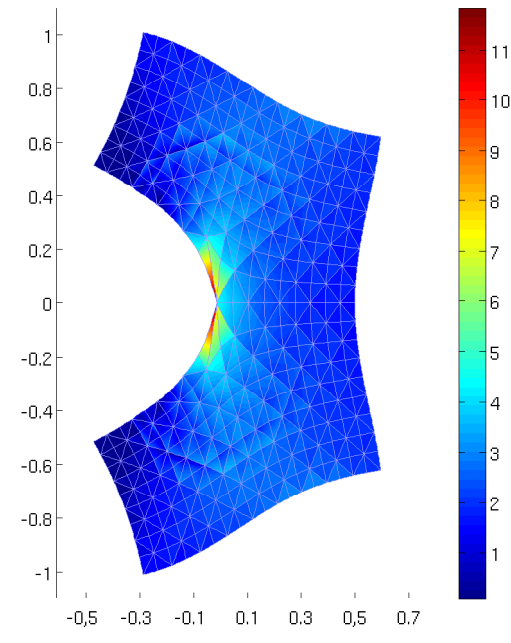

Figure 5: Von-Mises stress distribution (Blatz-Ko law, $\mathrm{h}=1 / 18$ )

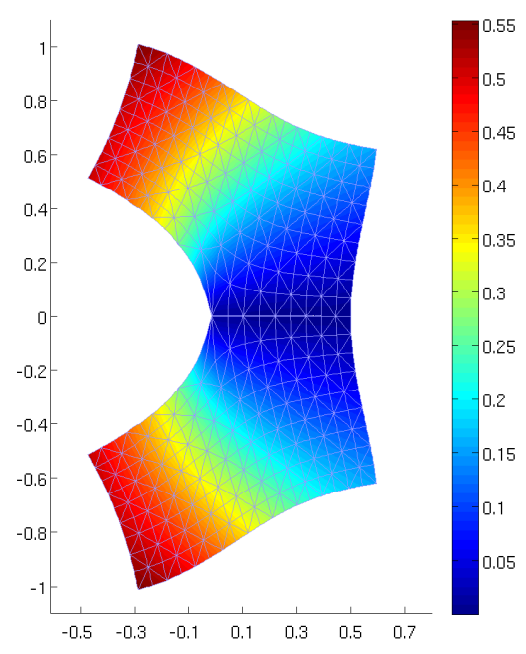

Figure 6: Displacement field distribution (Blatz-Ko law, $\mathrm{h}=1 / 18$ )

\subsection{Numerical study of singularity exponent}

The first step of the present analysis, is to verify numerically results given by the previous asymptotic procedure. The main idea is to find solution corresponding to the minimum of the system total potential energy $E_{p}$, whose expression is given by 1.11. Hence, the potential energy is computed as a function of the singularity exponent $\alpha$ by means of the generic finite element $\mathrm{C}++$ library Getfem $++^{1}$, preprogrammed to allow such operation (a general idea about Xfem codes implementation and a free $\mathrm{C}++$ based Xfem library are given in [5]), and for a transformation with the following representation:

$$
y_{2}(r, \theta)=r^{\alpha} f(\theta) \text {. }
$$

Let us note here, that there exist other numerical methods to determine singularities, such as the singularity exponent estimation based on classical finite element [28] and the adaptive singular element method, proposed in [11] for linear problems and neo-Hookean materials at large strain.

\footnotetext{
${ }^{1}$ http://download.gna.org/getfem/html/homepage/
} 


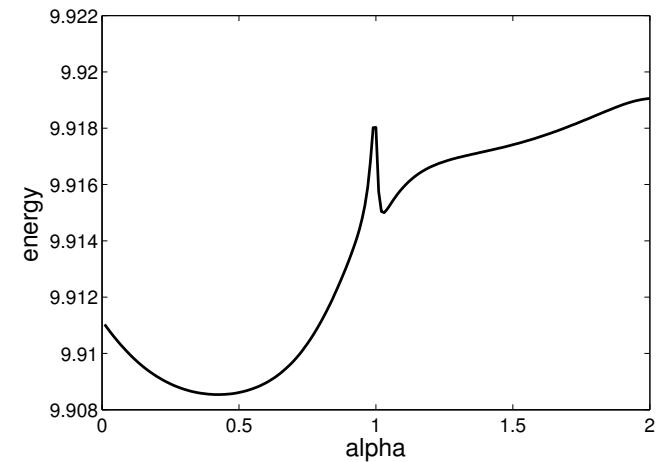

Figure 7: Energy as a function of singularity exponent (Blatz-Ko law, $h=1 / 20$ )

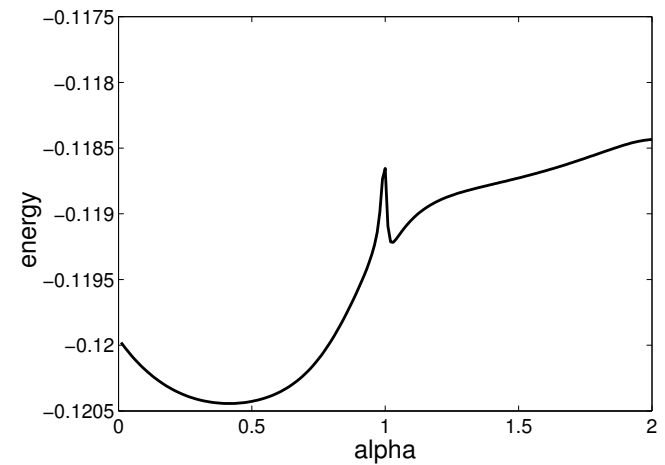

Figure 8: Energy as a function of singularity exponent (Ciarlet-Geymonat law, $h=1 / 20)$

Since the explicit form of the function $f(\theta)$ is supposed here unknown, we consider that it is sufficiently smooth, and we proceed to its decomposition into Fourier series:

$$
f(\theta)=\sum_{i} \beta_{i}^{1} \cos \left(i \frac{\theta}{2}\right)+\beta_{i}^{2} \sin \left(i \frac{\theta}{2}\right) .
$$

Taking into account the rapid convergence of this series, we keep only a few terms for the implementation. The enrichment space is then reduced to

$$
\left\{F_{i}\right\}=\left\{r^{\alpha} \cos \left(i \frac{\theta}{2}\right), r^{\alpha} \sin \left(i \frac{\theta}{2}\right)\right\} ; \quad i=1 . .7
$$

Figures 7 and 8 show that potential energy first minimum corresponds to a value of the singularity exponent close to the theoretical one (equal to $1 / 2$ ). For Blatz-Ko case, the hardening parameter $n$ was taken equal to 1. Since singularity exponent depends on this parameter, other values of $n$ was considered to verify the pertinence of results enunciated by (1.52). For $n=0.8(\alpha=0.375)$ and $n=2(\alpha=0.75)$, figures 9 and 10 confirm analytical predictions.

One can remark from figures 7 and 8 that the estimate of the minimum is not very accurate since the variations near the minimum are small. A consequence is that a small variation of the singularity exponent $\alpha$ is incapable to change remarkably the solution. An investigation of the influence of singularity exponent variation on convergence and approximation error will be presented in the next section.

Still in figures 7 and 8, a second minimum near $\alpha=1$ appears, but it is not necessary to take it into consideration for enrichment, since the classical 


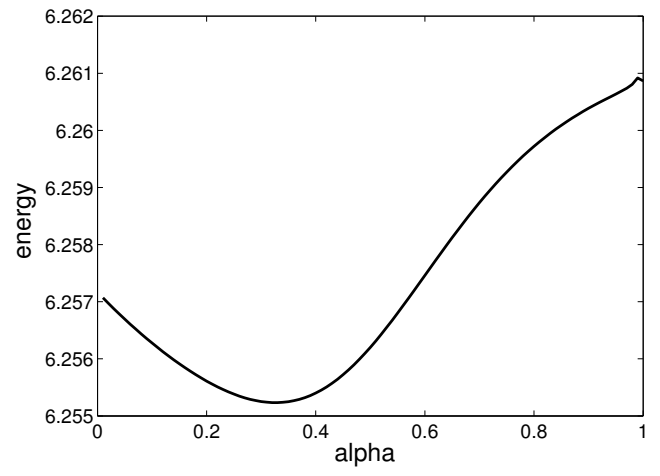

Figure 9: Energy as a function of singularity exponent (Blatz-Ko law, $n=0.8$, $h=1 / 20)$

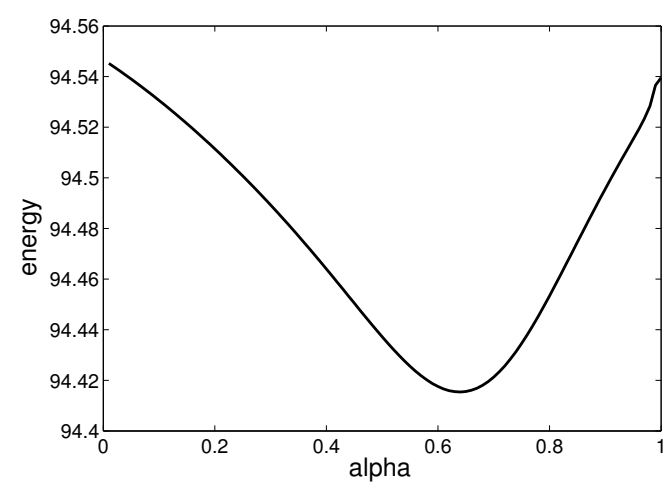

Figure 10: Energy as a function of singularity exponent (Blatz-Ko law, $n=2$, $h=1 / 20)$

finite element shape function can approximate a term $r^{1}$ in an optimal way. In the presented case, the mesh is rather coarse, which may explain the presence of this minimum. Indeed, Figure 11 proves that it disappears when mesh is slightly refined $(h=1 / 40)$, without the need of an extreme refinement. This does not mean that the minimum depends on mesh size because it is a very special case.

The same test investigates the existence of higher singularities. Thus, it consists to fix a first enrichment based on $r^{1 / 2}$, then checking if any singularity arises for $\alpha>1$. Nevertheless, the method fails to detect any minimum as shown in Figure 11.

\subsection{Convergence study}

In order to estimate the contribution of the Xfem enrichment, some convergence tests were established to compare the error in $L^{2}$ and $H^{1}$-norms found with the classical finite element method and those relative to the Xfem Cut-off. First, a Lagrange elements $P_{1}$ were used for both large strain and small strain cases. Figures 12, 13, 16 and 17 present convergence curves for the Blatz-Ko case, and Figures 14,15, 18 and 19 are associated to the Ciarlet-Geymonat case.

A convergence study is also made for $P_{2}$ elements, and enrichment was limited to the first singularity expression, since numerical study failed to detect higher terms (Figure 11). For both two potentials, results are given by Figures 20, 21, 22 and 23 .

Tables $(1,2)$ summarize main results of the convergence study for studied potentials. Now, let us recall that the function $r^{\frac{1}{2}} f(\theta)$ belongs to $\boldsymbol{H}^{3 / 2-\eta}(\Omega)$, 


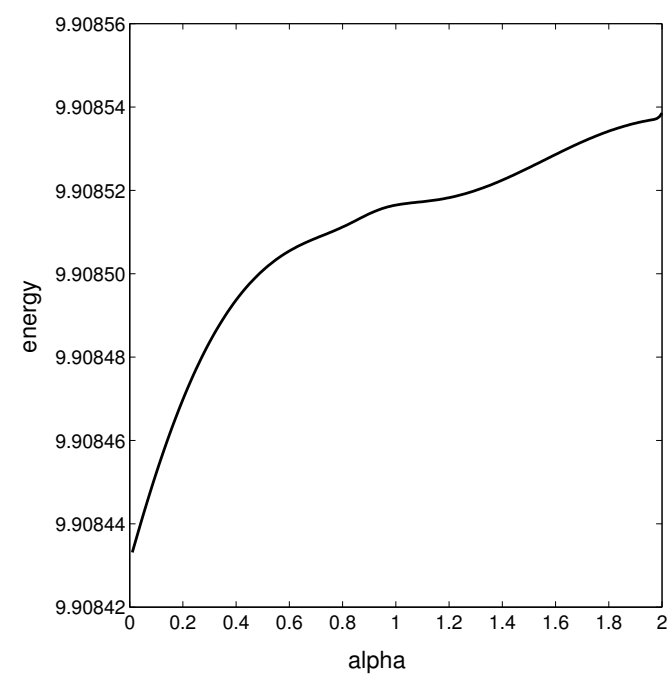

Figure 11: Energy as a function of singularity exponent (Blatz-Ko law, $h=$ $1 / 40)$, with fixed enrichment $r^{1 / 2}$

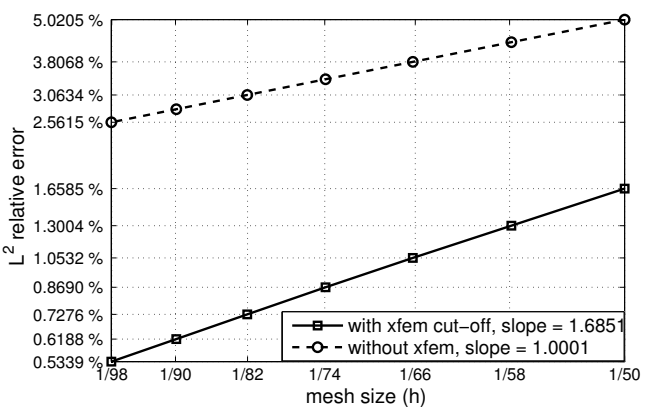

Figure 12: $L^{2}$-error for classical fem and Xfem cut-off, with $P_{1}$ elements (Blatz-Ko law), with small deformations condition

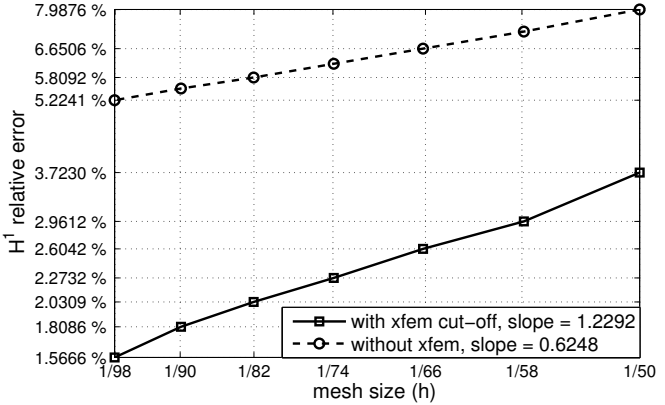

Figure 13: $H^{1}$-error for classical fem and Xfem cut-off, with $P_{1}$ elements (Blatz-Ko law), with small deformations condition 


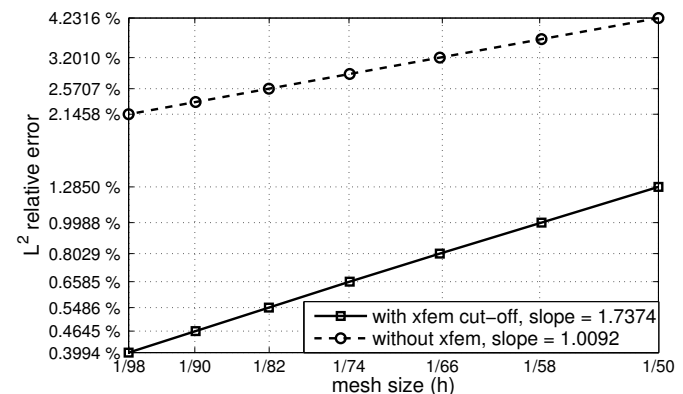

Figure 14: $L^{2}$-error for classical fem and Xfem cut-off, with $P_{1}$ elements (CiarletGeymonat law), with small deformations condition

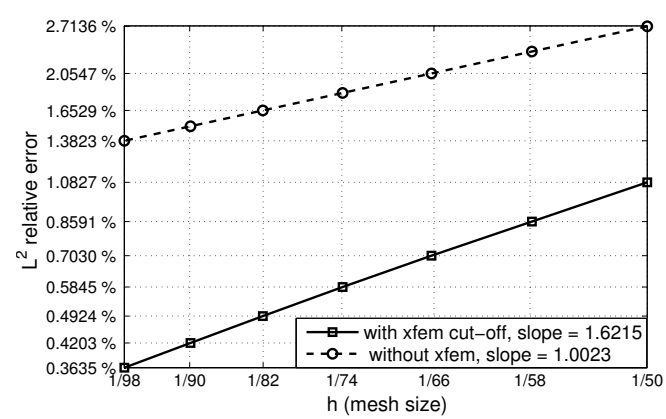

Figure 16: $L^{2}$-error for classical fem and Xfem cut-off, with $P_{1}$ elements (Blatz-Ko law)

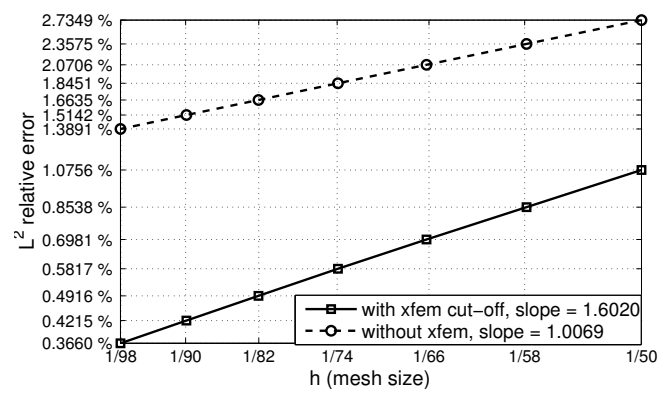

Figure 18: $L^{2}$-error for classical fem and Xfem cut-off, with $P_{1}$ elements (CiarletGeymonat law)

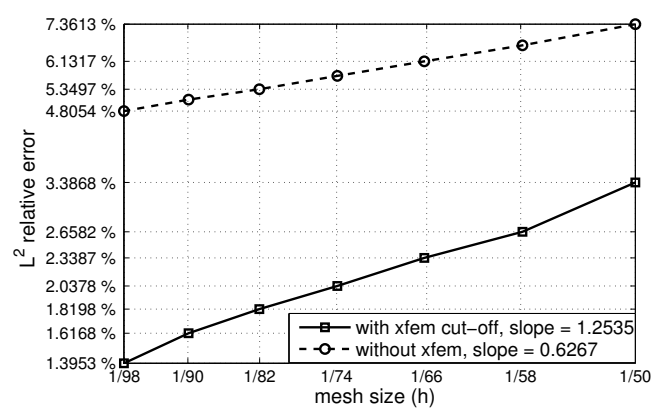

Figure 15: $H^{1}$-error for classical fem and Xfem cut-off, with $P_{1}$ elements (CiarletGeymonat), with small deformations condition

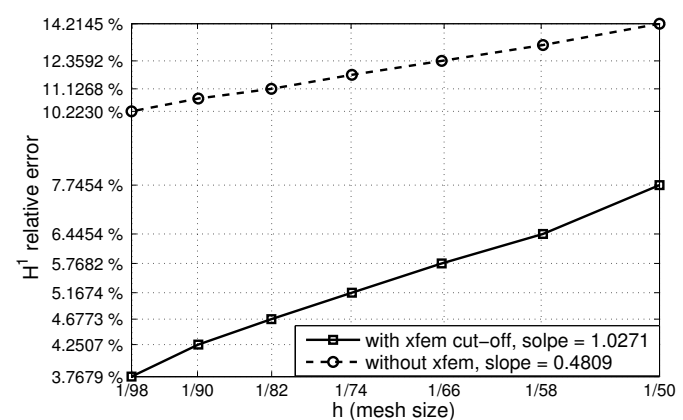

Figure 17: $H^{1}$-error for classical fem and Xfem cut-off, with $P_{1}$ elements (Blatz-Ko law)

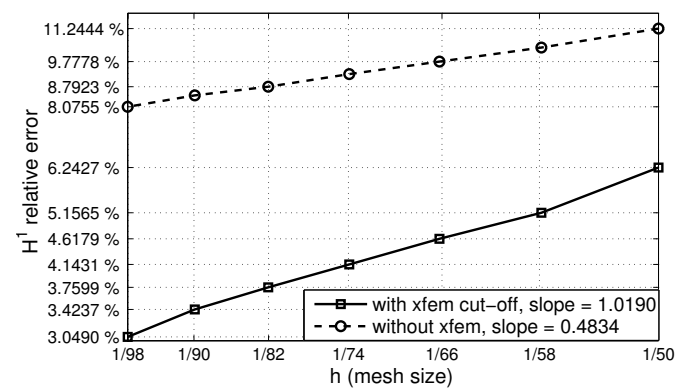

Figure 19: $H^{1}$-error for classical fem and Xfem cut-off, with $P_{1}$ elements (CiarletGeymonat) 


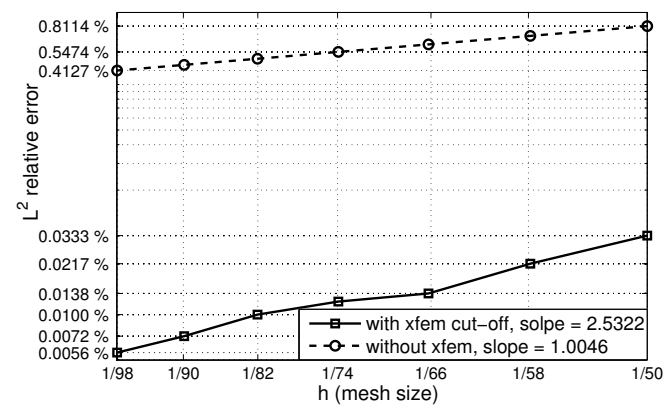

Figure 20: $L^{2}$-error for classical fem and Xfem cut-off, with $P_{2}$ elements (Blatz-Ko)

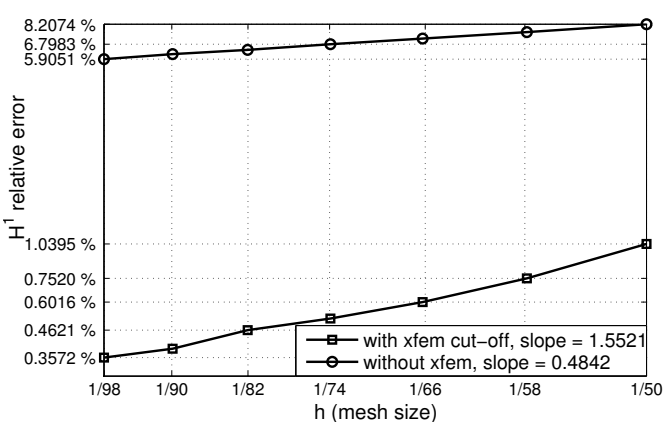

Figure 21: $H^{1}$-error for classical fem and Xfem cut-off, with $P_{2}$ elements (Blatz-Ko)

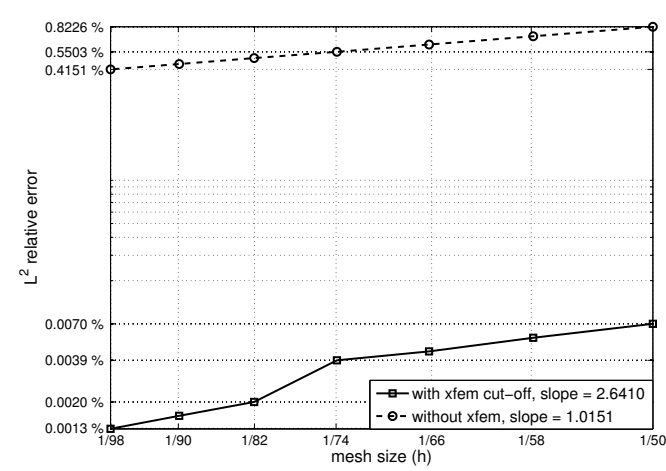

Figure 22: $L^{2}$-error for classical fem and Xfem cut-off, with $P_{2}$ elements (CiarletGeymonat law)

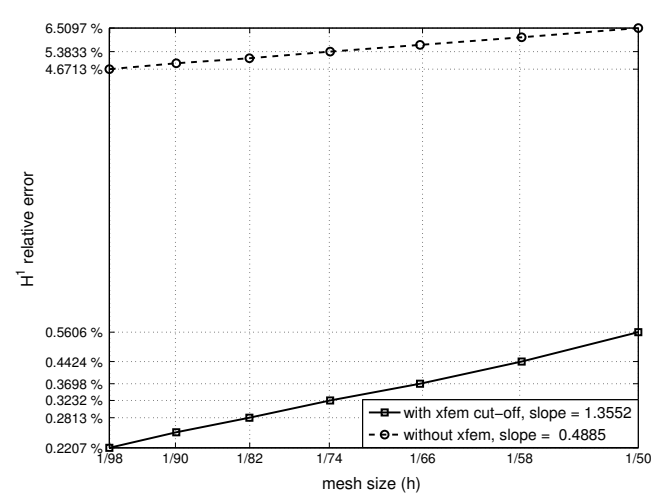

Figure 23: $H^{1}$-error for classical fem and Xfem cut-off, with $P_{2}$ elements (CiarletGeymonat) 
$\forall \eta>0$ (see [15] for the linear case). Consequently, the convergence of classical finite element method is limited to $O\left(h^{1 / 2}\right)$ for the norm of energy (and $O(h)$ for the $L^{2}$-norm). This was confirmed through all tests realized with this method and independently of the type of used elements $\left(P_{1}\right.$ or $\left.P_{2}\right)$.

It was proved in [26] that this Xfem variant gives an optimal convergence rate for linear problems. However, there is no work in literature generalizing this result for nonlinear problems. Consequently, it is not guaranteed to find an optimal convergence in such a case. Nevertheless, optimality was attained for the norm of energy and for $P_{1}$ elements. A considerable improvement is noticed for the $L^{2}$-norm of the errors, which decrease considerably with the application of the Xfem method. An analogous observation is made for the small deformations case, with better results for the $L^{2}$-norm and a rapid convergence of the $H^{1}$-norm (slightly over optimal).

Concerning $P_{2}$ elements, they lead to a relative optimality, if we suppose that the next term of the asymptotic development is of the order of $r^{3 / 2}$ (as given theoretically). Indeed, the best convergence reachable rate in this case is limited to $h^{3 / 2}$ for $H^{1}$-norm and $h^{5 / 2}$ for $L^{2}$-norm, which was found through realized tests.

\begin{tabular}{|l||l|l||l|l|}
\hline \multicolumn{1}{|l||}{ Elements } & \multicolumn{2}{c||}{$P_{1}$} & \multicolumn{2}{c|}{$P_{2}$} \\
\hline Norm & $\|\cdot\|_{L_{2}}$ & $\|\cdot\|_{H_{1}}$ & $\|\cdot\|_{L_{2}}$ & $\|\cdot\|_{H_{1}}$ \\
\hline classical fem & 1.0023 & 0.4809 & 1.0046 & 0.4852 \\
\hline xfem cut-off & 1.6215 & 1.0271 & 2.5322 & 1.5521 \\
\hline
\end{tabular}

Table 1: fem and xfem cut-off convergence rates for Blatz-Ko potential

\begin{tabular}{|l||l|l||l|l|}
\hline \multicolumn{1}{|l||}{ Elements } & \multicolumn{2}{c||}{$P_{1}$} & \multicolumn{2}{c|}{$P_{2}$} \\
\hline Norm & $\|\cdot\|_{L_{2}}$ & $\|\cdot\|_{H_{1}}$ & $\|\cdot\|_{L_{2}}$ & $\|\cdot\|_{H_{1}}$ \\
\hline classical fem & 1.0069 & 0.4834 & 1.0151 & 0.4885 \\
\hline xfem cut-off & 1.6020 & 1.0190 & 2.6410 & 1.3552 \\
\hline
\end{tabular}

Table 2: fem and xfem cut-off convergence rates for Ciarlet-Geymonat potential

In order to verify the influence of the variation of the singularity exponent in the Xfem enrichment, two tests were realized. The first one (Figure 24) consists in looking into effects of small variation of $\alpha$ on convergence and approximation error (only the $H^{1}$-norm test are presented, since the $L^{2}$ norm test leads to the same conclusion). The second test look into the effects of large variations of $\alpha$ (Figures 25 and 26). In order to make comparison 


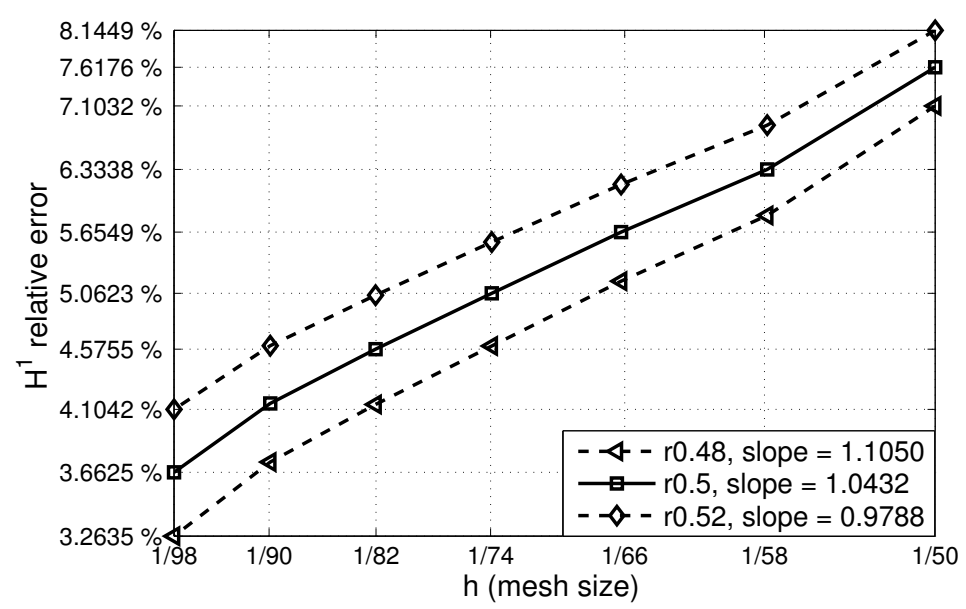

Figure 24: Influence of small variations of the singularity exponent on the $H^{1}$-norm (Blatz-Ko law)

between all obtained curves coherent, same conditions were guaranteed for all cases and the enrichment basis is the one given by (3.3), even when $\alpha=0.5$.

Contrary to the second minimum, the first one is smooth enough to make solution unchanged for a small variation of $\alpha$, as illustrated through Figure 24. Figures 25 and 26 show the influence of an important variation of the parameter $\alpha$ on the convergence of the Xfem method. We remark that the best convergence rate is obtained for $\alpha=0.4$ and $\alpha=0.5$, due to the fact that they minimize energy more than others. Besides, the corresponding convergence curves keep a constant slope, contrary to other ones $(\alpha=0.2$, $\alpha=0.3)$ for which, slopes degrade when mesh is refined. This is probably due to the underestimation of the singularity for unrefined mesh. Indeed, when this last is more precise, the estimated value of $\alpha$ increases more and more, which makes the consideration of $\alpha=0.2$ and $\alpha=0.3$, more and more erroneous.

An other important notice is seen through previous tests. The comparison between solutions obtained from the analytical and the serial form of the enrichment for the case when $\alpha=0.5$, shows that results are similar, and leads consequently to the coherence of assumption made in (3.3).

A concluding remark about improvement obtained with the Xfem cutoff, is that this method improves results without increasing the number of degrees of freedom. Table (3), compares the number of degrees of freedom used by the classical finite element method and Xfem cut-off variant, and shows that it is almost the same. 


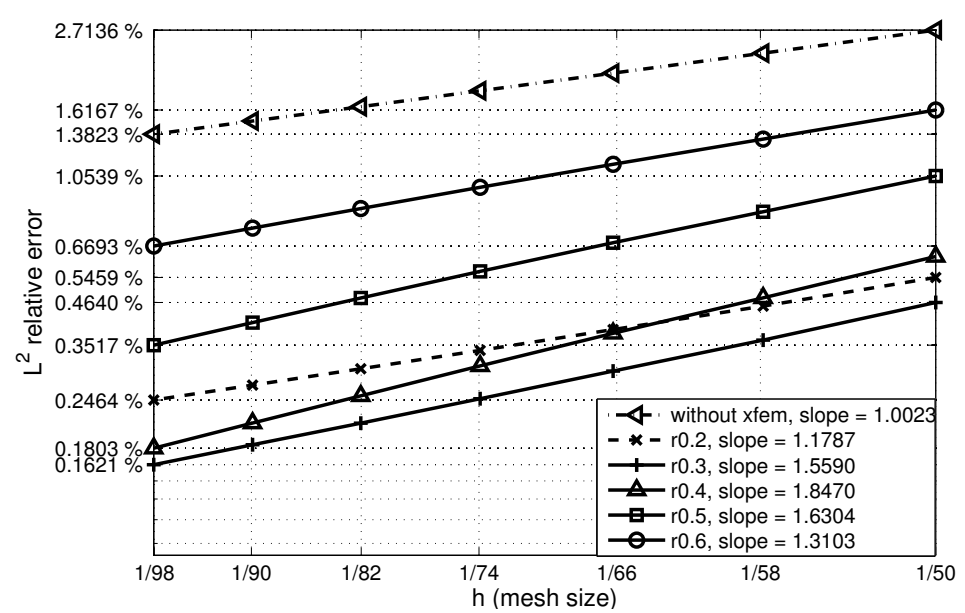

Figure 25: Influence of considerable variations of the singularity exponent on the $L^{2}$-norm (Blatz-Ko law)

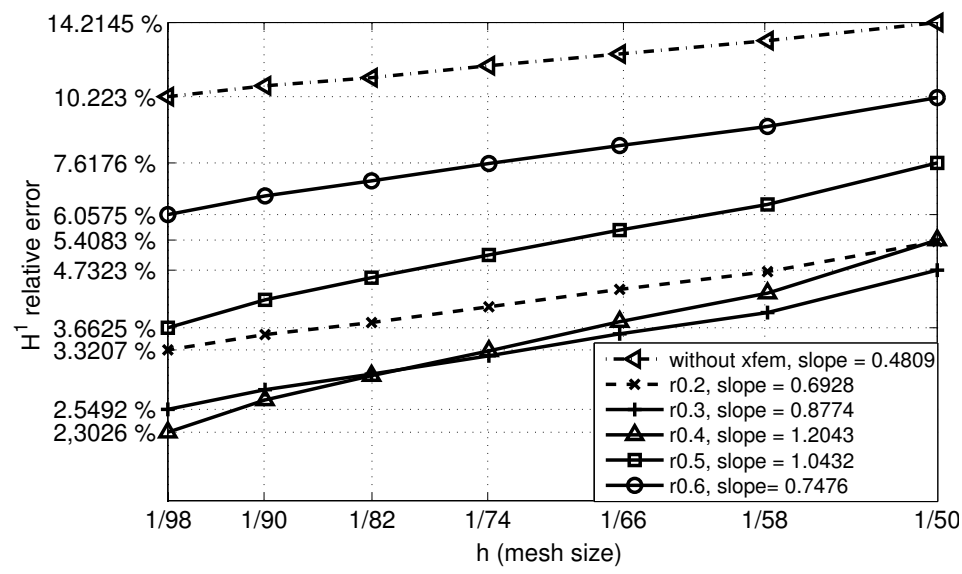

Figure 26: Influence of considerable variations of the singularity exponent on the $H^{1}$-norm (Blatz-Ko law) 


\begin{tabular}{|c||c|c|}
\hline Number of cells in each direction & Classical fem & Xfem Cut-off \\
\hline 26 & 1487 & 1489 \\
\hline 50 & 5255 & 5257 \\
\hline 90 & 16655 & 16657 \\
\hline
\end{tabular}

Table 3: Number of degrees of freedom of classical fem and Xfem (Blatz-Ko potential)

\section{Conclusion}

In the present paper, an analysis of a singular problem in cracked domain was carried out. The study deals with the fully nonlinear theory at large strain, and aims to apply the Xfem method in order to overcome the limitations of classical finite element method, when used for such cases. In spite of the absence of analytical and mathematical proofs, results were relevant (analogous to linear theory predictions) and emphasize the contribution of the Xfem cut-off variant to the improvement of numerical convergence and estimation errors, without deteriorating the linear system conditioning or increasing numerical problem size. Besides, the established work, leads to a coherence between results obtained from the asymptotic procedure and those obtained numerically, since each one confirms other. Finally, we should keep in mind an interesting observation concerning the smooth character of the first minimum of potential energy. Indeed, this proves that even a non-precise estimation of the first singularity exponent does not affect considerably the approximated solution.

\section{References}

[1] M. Baker and J.L. Ericksen. Inequalities restricting the form of the stressdeformation relations for isotropic elastic solids and reiner-rivlin fluids. $J$. Wash. Acad. Sci, 44:33-35, 1954.

[2] J. M. Ball. Convexity conditions and existence theorems in nonlinear elasticity. Archive for rational mechanics and Analysis, 63(4):337-403, 1976.

[3] E. Béchet, H. Minnebo, N. Moës, and B. Burgardt. Improved implementation and robustness study of the $\mathrm{x}$-fem for stress analysis around cracks. Int. J. Numer. Meth. Engng, 64:1033-1056, 2005.

[4] P.J. Blatz and W.L. Ko. Application of finite elastic theory to the deformation of rubbery materials. Trans. Soc. Rheol, 6:223-251, 1962. 
[5] P. V. Bordas, S.and Nguyen, C. Dunant, A. Guidoum, and H. Nguyen-Dang. An extended finite element library. Int. J. Numer. Meth. Engng, 71:703-732, 2007.

[6] E. Chahine, P. Laborde, and Y. Renard. Crack tip enrichment in the xfem method using a cut-off function. Int. J. Numer. Meth Engng, 75:629-646, 2008 .

[7] N. Chevaugeon, N. Moës, and H. Minnebo. Improved crack tip enrichment functions and integration for crack modeling using the extended finite element method. International Journal for Multiscale Computational Engineering, 11:597-631, 2013.

[8] P.G. Ciarlet. Mathematical Elasticity, Volume 1: Three-Dimensional Elasticity. North-Holland, 1988.

[9] B. D. Coleman and W. Noll. On the thermostatics of continuous media. Archive for rational mechanics and analysis, 4(1):97-128, 1959.

[10] B. D. Coleman and W. Noll. Material symmetry and thermostatic inequalities in finite elastic deformations. Archive for Rational Mechanics and Analysis, 15(2):87-111, 1964 .

[11] R. Denzer, M. Scherer, and P. Steinmann. An adaptative singular finite element in nonlinear fracture mechanics. International Journal of Fracture, 147:181-190, 2007.

[12] T. Elguedj, A. Gravouil, and A. Combescure. Appropriate extended functions for x-fem simulation of plastic fracture mechanics. Comput. Methods Appl. Mech. Engrg, 195:501-515, 2006.

[13] T.P. Fries and T. Belytschko. The extended/generalized finite element method: An overview of the method and its applications. Int. J. Numer. Meth. Engng, 84:253-304, 2010.

[14] G. Geymonat and P. G. Ciarlet. Sur les lois de comportement en élasticité non linéaire compressible. CR Acad. Sci. Paris Sér. II, 295:423-426, 1982.

[15] P. Grisvard. Singularities in boundary value problems. Masson, 1992.

[16] A.R. Khoei, S.O.R. Biabanaki, and M. Anahid. Extended finite element method for three-dimensional large plasticity deformations on arbitrary interfaces. Comput. Methods Appl. Mech. Engrg, 197:1100-1114, 2008.

[17] A.R. Khoei, S.O.R. Biabanaki, and M. Anahid. A lagrangian-extended finiteelement method in modeling large-plasticity deformations and contact problems. International Journal of Mechanical Sciences, 51:384-401, 2009. 
[18] J. K. Knowles and E. Sternberg. Finite-deformation analysis of the elastostatic field near the tip of a crack: Reconsideration and higher-order results. Journal of Elasticity, 4(3):201-233, 1974.

[19] J.K. Knowles and E. Sternberg. An asymptotic finite-deformation analysis of the elastostatic field near the tip of a crack. Journal of Elasticity, 3(2):67-107, 1973.

[20] K.C. Le and H. Stumpf. The singular elastostatic field due to a crack in rubberlike materials. Journal of elasticity, 32:183-222, 1993.

[21] G. Legrain, N. Moës, and E. Verron. Stress analysis around crack tips in finite strain problems using the extended finite element method. Int. J. Numer. Meth. Engng, 63:290-314, 2005.

[22] S. Loehnert, D.S. Mueller-Hoeppe, and P. Wriggers. 3d corrected xfem approach and extension to finite deformation theory. Int. J. Numer. Meth. Engng, 86:431-452, 2011.

[23] N. Moës and T. Belytschko. X-fem: de nouvelles frontières pour les éléments finis. Revue européenne des éléments finis, 11:131-150, 1999.

[24] N. Moës, J. Dolbow, and T. Belytschko. A finite element method for crack growth without remeshing. Int. J. Numer. Meth. Engng, 46:131-150, 1999.

[25] S. Mohammadi. Extended finite element method. Blackwell Publishing, 2008.

[26] S. Nicaise, Y. Renard, and E. Chahine. Optimal convergence analysis for the extended finite element method. Int. J. Numer. Meth. Engng, 86:528-548, 2011.

[27] R.W. Ogden and D.A. Isherwood. Solution of some finite plane-strain problems for compressible elastic solids. The Quarterly Journal of Mechanics and Applied Mathematics, 31(2):219-249, 1978.

[28] G.H. Staab. Estimating singularity powers with finite elements. Computers and Structures, 17:73-78, 1983.

[29] R.A. Stephenson. The equilibrium field near the tip of a crack for finite plane strain of incompressible elastic materials. Journal of elasticity, 12(1):65-99, 1982.

[30] M. Stolarska, D. L. Chopp, N. Moës, and T. Belytschko. Modelling crack growth by level sets in the extended finite element method. Int. J. Numer. Meth. Engng, 51:943-960, 2001.

[31] A.M. Tarantino. Thin hyperelastic sheets of compressible material: Field equations, airy stress function and an application in fracture mechanics. Journal of Elasticity, 44:37-59, 1996. 